Method Semi-structured interviews were conducted with registered nurses working in hospital $(n=6)$ and hospice $(n=29)$ settings. The roles and experiences of nurses when meeting the psychological needs of patients receiving palliative care were explored. Interviews were audio recorded, transcribed verbatim and analysed thematically.

Result Four themes were generated after analysis of the data collected. These are: assessing, observing, intuition and experience; trust and managing uncertainties; little things that have great impacts; maintaining professionalism when grieving.

Conclusion Nurses are well placed to detect, assess and manage psychological problems experienced by patients receiving palliative care, with frequent contact being a key factor. Nurses are faced with emotional stress when caring for patients at the end of life and coping mechanisms and strategies are needed to enable them to continue to function well as a compassionate carer.

\section{AUDIT OF THE PHARMACOLOGICAL MANAGEMENT OF DEPRESSION IN PALLIATIVE CARE}

Catriona Barrett-Ayres, Helen Jones, Hannah Clare, Sophie Harrison, Julie Suman, Denise Brady. North West Audit Group

10.1136/spcare-2020-PCC.127

Background Depression management in palliative care is challenging. The North West Palliative Care Audit Group (NWAG) coordinated a review revealing poor adherence to guidelines. Participating organisations were therefore asked to develop action plans.

Aim To audit the pharmacological management of depression against evidence-based standards (references 1-5).

Methods A multi-centre retrospective case note review of patients who were commenced antidepressants for depression was undertaken. $90 \%$ compliance was deemed acceptable. Four areas were audited: assessment and diagnosis, communication, follow up and information sharing.

Results Seven hospices and three hospital teams returned 58 forms. Mirtazapine was used in $67 \%$, citalopram in $19 \%$ and sertraline in $11 \%$. Only two sub-standards out of a total of 42 achieved compliance. These were regarding concomitant use of a benzodiazepine or a review of medication where a patient becomes agitated on initiation of antidepressant and also regarding sharing the diagnosis when a patient changed healthcare setting. Particular areas requiring improvement were documentation of: the duration of episode $(50 \%)$, the severity (21\%), functional impact $(57 \%)$, previous episodes (30\%); medications used (17\%); contributory causes (76\%); alternative diagnoses (34\%); suicidal ideation $(22 \%)$ and poor communication to patients. Only $72 \%$ were documented to have an explanation of the concept of depression, 59\% regarding non-pharmacological treatments, 50\% regarding side effects and 29\% the lag in effect. No patients were offered written information. There was poor documentation of follow up and hand over to health care professionals.

Conclusion Practice was poor in all areas except two. Findings support the need for education of staff regarding diagnosis, communication, follow up and information sharing. In particular: documentation of suicidal ideation (22\%); severity (21\%); past history $(30 \%)$ and provision of written information to patients $(0 \%)$ was poor. Recommendations were made to run educational sessions and a depression prompt was devised for local adaptation. A re-audit is planned. (References: 1-5 supplied on poster).

\section{LONELINESS IS COMMON AMONG HONG KONG CHINESE CANCER PATIENTS RECEIVING PALLIATIVE CARE}

${ }^{1}$ Raymond KW Woo, ${ }^{2}$ Wai-him Cheung, ${ }^{1}$ Clara Y Poon, ${ }^{1}$ Annie OL Kwok. ${ }^{1}$ Caritas Medical Centre; ${ }^{2}$ Dimension Center

\subsection{6/spcare-2020-PCC.128}

Background The negative health impact of loneliness is increasingly recognized, however, its prevalence among cancer patients receiving palliative care is yet unknown.

Methods This is a cross-sectional study performed in the palliative care unit of Caritas Medical Centre, Hong Kong. Patients were invited to response the single-item questions, 'Are you feeling lonely? (Yes or no.)' Loneliness severity was assessed using the local version of De Jong Gierveld Loneliness Scale (short form). Symptom burden was assessed by the local version of the Edmonton Symptom Assessment Scale (ESAS) with the 10th item assigned as 'most lonely'. Patient's demographic and health-related data were extracted thru interview and health record.

Results Fifty-six patients participated in the study, the mean age of participants was 72.7 years old. The median Palliative Performance Scale was 70. Eight patients had a concomitant psychiatric illness. Seventeen patients (30.4\%) answered 'yes' on the single-item question of loneliness. Eleven and ten patients were scored as severe loneliness in the emotional and social loneliness subscale of De Jong Gierveld Loneliness scale respectively. For those who admitted themselves lonely in the single-item questions, they scored higher in ESAS-Depression (5 vs 0 ), Anxiety (5 vs 0), Impaired Well-Being (5 vs 3 ) and Loneliness subscale ( 7 vs 0 ) as well as HADS-Depression (13 vs 8 ), -Anxiety (9 vs 4) and Total Score (22 vs 12). (MannWhitney $U$ test, <0.05). Half of the patients rated 0 out of 10 in the ESAS loneliness subscale, while 21 patients rated 5 or above. It was correlated with HADS-Depression, -Anxiety and -Total score (Spearman correlation, $\mathrm{p}<0.001$ ).

Conclusions Loneliness is common among Hong Kong cancer patients receiving palliative care. The severity of loneliness may correlate with the severity of depression and anxiety.

\section{Service development | posters $109-163$}

\section{UNDER THE INFLUENCE? THE HIDDEN DRUG DRIVING CONCERNS}

Maimoona Ali. Sheffield Teaching Hospitals NHS Foundation Trust

\subsection{6/spcare-2020-PCC.129}

Background The implications of the drug driving guidance on our specialist palliative care unit is unknown. We make assumptions that patients don't drive a vehicle. It's not a routine question on admission or something that is addressed whilst inpatient medication changes are being made and also not something discussed when a patient is discharged.

Methods A snapshot assessment of the 18 patients admitted to the inpatient specialist palliative care unit was performed on 